\title{
Disposição geológica de rejeitos radioativos no Brasil: diretrizes de proteção radiológica, requisitos e avaliação de segurança
}

GeOLOGICAL disPosal OF RADIOACTIVE WASte In BRAZIL: GUIDELINES FOR RADIOLOGICAL PROTECTION, REQUIREMENTS AND SAFETY ASSESSMeNt

Paulo Fernando Lavalle Hellbron Filho ${ }^{1}$, Corbiniano Silva², Rex Nazaré Alves³, Ana Maria Xavier², Mônica Pereira Lavalle Hellbron² e Claudio de Morisson VaLeRIANO ${ }^{2}$

1 - Comissão Nacional de Energia Nuclear. Coordenação de Rejeitos. RJ, Brasil. E-mall: paulo@cnen.gov.br; axavier@cnen.gov.br,

2 - Univ. do Estado do Rio de Janeiro. Facul.e de Geologia. RJ, Brasil. E-mall: Corbiniano@gmall.com; monica.hellbron@gmall.com;valeriano.claudi@@gmall.com

3 - Instituto Militar de Engenharia. RJ, Brasil E-mall: rexnazare@ime.eb.br

Abstract: High-level radioactive waste and its safe final disposal is a complex problem; a few countries have managed to provide an adequate solution for it, since it contains highly radioactive materials that result from reactions occurring within nuclear reactors. Spent nuclear fuel may be considered a waste because it is no longer efficient in generating electricity due to decrease of the fission process. However, it is still thermally hot, highly radioactive, potentially harmful and must be handled and stored with care. Since the only natural way high level waste can lose their radioactive contents is through decay, hundreds of thousands of years would be necessary to become harmless, from a radiological point of view. Thus, to provide adequate protection of the public and environment for such long period of time, the only alternative for permanent disposal of this type of waste is a geological repository, whose complexity for site selection, construction and environmental licensing, among other aspects, is enormous. According to the International Atomic Energy Agency (IAEA), geological repositories are considered the main and most suitable solution for disposal of high level radioactive waste that remains dangerous for millions of years and needs to be completely isolated from biosphere. Therefore, this article, based on IAEA documents as well as on Brazilian Nuclear Energy Commission (CNEN) requirements, points out the main aspects that should be considered for the licensing of geological disposal facilities in the country, including the most important aspects of radiation protection and other elements that must be taken into account

Manuscrito:

Recebido: 25/01/18

Corrigido: $8 / 08 / 18$

Aceito: $11 / 08 / 18$

Citation:Heilbron Filho P.F.L., Silva C., Alves R.N, Xavier A.M., Heilbron M.P.L, Valeriano C.M.. 2018. Disposição geológica de rejeitos radioativos no Brasil: diretrizes de proteção radiológica, requisitos e avaliação de segurança. Terræ Didatica, 14(3):196-206. URL: http://www.ige.unicamp.br/terraedidatica/.

Keywords: Nuclear, high-level radioactive waste, geological sites, guidelines. during site selection, planning, construction, operation and closure of geological repositories.

\section{Introdução}

A questão da deposição dos rejeitos radioativos de alto nível de radiação tem sido um processo complicado em todo o mundo, sobretudo pelos custos e recursos humanos envolvidos e necessários para localizar sítios adequados para armazenarem por milhares de anos este tipo de passivo, considerado altamente perigoso, cujos impactos ambientais podem ser catastróficos a longo prazo caso não sejam adequadamente isolados da biosfera. No caso brasileiro, que atualmente possui 2 usinas nucleares e uma em construção, a preocupação com os rejeitos nucleares de alto nível é ainda incipiente. Entretanto, é fundamental que a CNEN inicie estudos visando o desenvolvimento de metodologias que possam contribuir para a implementação de políticas, normas e regulamentações no sentido de viabilizar o planejamento adequado de ações para a construção de um depósito geológico no Brasil com vistas a abrigar os rejeitos radioativos de alto nível provenientes das usinas nucleares (Silva et al. 2015).

Do ponto de vista normativo, a CNEN possui apenas uma norma para o licenciamento de depósitos de rejeitos para baixo nível de radiação (CNEN 2014), não havendo nenhuma outra voltada para a deposição dos rejeitos radioativos de alto nível, os quais exigem a colocação dos mesmos em grandes profundidades (da ordem de centena de metros), chamados repositórios geológicos, segundo IAEA (1977, 1989, 1994, 2006, 2011).

A deposição final dos rejeitos radioativos é uma atividade que deve ser regulamentada, visando garantir o confinamento seguro desses materiais, pelo tempo que se fizer necessário, alcançando 3 objetivos primordiais: (i) A minimização de qualquer efeito nocivo às gerações futuras; (ii) A proteção à saúde humana; e (III) a proteção ao meio ambiente. Assim, é de fundamental impor- 
tância que os requisitos de segurança necessários ao desenvolvimento de repositórios para rejeitos de alto nível sejam estabelecidos pelos governos dos países onde existam aplicações para a energia nuclear, em especial reatores nucleares.

No caso do Brasil, compete à CNEN, tanto a função de receber e depositar rejeitos radioativos como a de avaliar a segurança e proceder à fiscalização dos repositórios. O órgão brasileiro responsável pelo licenciamento ambiental de repositórios, o IBAMA, exige nesses casos a elaboração de Estudos e Relatórios de Impacto do Meio Ambiente (EIA-RIMA). O EIA deve ser elaborado pelo operador do depósito que inclua todas as análises necessárias para se estimar as prováveis consequências do repositório e como ele pode afetar negativamente o meio ambiente. O RIMA é um relatório conclusivo que traduz os termos técnicos do EIA para uma linguagem mais simples, visando o esclarecimento da população quanto dos possíveis impactos ambientais do empreendimento. Este relatório é responsável pelos levantamentos e conclusões para que o órgão público licenciador faça uma análise no sentido de aceitar o licenciamento do projeto. O EIA e o RIMA são exigidos na fase de licença prévia do repositório.

Este artigo introduz a problemática que envolve a disposição segura dos rejeitos radioativos de alto nível, um dos gargalos do setor nuclear brasileiro, tendo em vista a construção de novas usinas, apontando os aspectos importantes relacionados com o licenciamento de repositórios geológicos. Nesta perspectiva, o documento aponta os principais aspectos de proteção radiológica, avaliação e requisitos de segurança, enquanto diretrizes que devem ser levadas em consideração quando do projeto e construção de um depósito geológico, tipo de depósito que deverá ser utilizado para a deposição segura dos rejeitos de alto nível oriundos dos combustíveis irradiados nos reatores nucleares das usinas de Angra dos Reis.

\section{A questão dos rejeitos radioativos}

Segundo a CNEN, rejeitos radioativos são "qualquer material resultante das atividades humanas, que contenha radionuclídeos em quantidades superiores aos limites de isenção especificados na norma CNEN-NE-6.02, e para o qual a utilização é imprópria ou imprevista". Em função desses materiais permanecerem radioativos por longos períodos, todo material radioativo que é considerado rejeito não isento deve ser recolhido, tratado e armazenado adequadamente em instalações especificas para essa finalidade, havendo a necessidade de serem acondicionados em depósitos especialmente construídos para este fim, chamados de repositórios.

\subsection{Classificação dos rejeitos radioativos}

De acordo com a IAEA, os rejeitos radioativos são classificados em três categorias (Tab. 1).

Os radioisótopos de vida curta consistem naqueles cujas meias-vidas são, no máximo, da ordem de 30 anos, casos do Cs-137, Sr-90, Kr-85, H-3, entre outros.

O estabelecimento de limites baseia-se no fato da radioatividade não ser um fenômeno recente: o homem sempre viveu em um mundo radioativo. O corpo humano é formado, também, por

Tabela 1. Classificação dos rejeitos radioativos. (IAEA 2009).

\begin{tabular}{|c|c|c|}
\hline Tipo de Rejeito & Características & Opção de Deposição \\
\hline 1. Isento & $\begin{array}{l}\text { Níveis de atividade igual ou inferior aos limites de } \\
\text { isenção que foram baseados em uma dose anual para os } \\
\text { membros do público inferior a } 0,01 \mathrm{mSv} \text {. }\end{array}$ & Sem restrição radiológica \\
\hline $\begin{array}{l}\text { 2. Baixo e Médio } \\
\text { Níveis }\end{array}$ & $\begin{array}{l}\text { Níveis de atividade superior ao limite de isenção e } \\
\text { geração de calor inferior ou da ordem } 2 \mathrm{~kW} / \mathrm{m}^{3} \text {. } \\
\text { Concentração de emissores alfa de meia vida longa } \\
\text { restrita (radionuclídeos igual ou inferior a } 4000 \mathrm{~Bq} / \mathrm{g} \\
\text { sendo que a média de todos os radionuclídeos no } \\
\text { embalado inferior a } 400 \mathrm{~Bq} / \mathrm{g} \text { ). }\end{array}$ & $\begin{array}{l}\text { Depósitos próximo à superfície } \\
\text { ou geológicos. }\end{array}$ \\
\hline 2.2. Vida Longa & $\begin{array}{l}\text { Concentração de radionuclídeos emissores alfa } \\
\text { superiores aos valores anteriores }\end{array}$ & Depósitos geológicos \\
\hline 3. Alto Nível & $\begin{array}{l}\text { Geração de calor superior a } 2 \mathrm{~kW} / \mathrm{m}^{3} \text { e concentração de } \\
\text { radionuclídeos emissores alfa superiores as estabelecidas } \\
\text { para os rejeitos de meia vida curta (item } 2.1 \text { ) }\end{array}$ & Depósitos geológicos \\
\hline (c) Terrae D & Campinas, SP & p. $196-206$ \\
\hline
\end{tabular}


elementos radioativos (C-14, K-40, etc) e a vida no planeta terra sempre foi sujeita às radiações provenientes dos raios cósmicos e dos elementos existentes na crosta terrestre, nas rochas, no solo, na água, nos alimentos e no ar. Esta radiação a que todos estamos sujeitos é conhecida como radiação de fundo.

Os rejeitos oriundos da operação de reatores (resinas, filtros, etc) são classificados na categoria 2.1. Os rejeitos oriundos de plantas de reprocessamento são classificados nas categorias 2.1 e 2.2. Os rejeitos oriundos das áreas médicas, industriais e de pesquisa são enquadrados na categoria 2.1. Os rejeitos resultantes de operações de descomissionamento são enquadrados nas categorias 2.1 e 2.2.

Os elementos combustíveis irradiados são classificados na categoria 3, considerados os mais perigosos, gerados durante a fissão do urânio nos reatores nucleares. Eles são bastante ativos e permanecem radioativos por um longo tempo, devendo ser segregados, imobilizados e abrigados em repositórios situados em grandes profundidades, centenas de metros da superfície, em terrenos geologicamente estáveis e selecionados para prover o isolamento adequado de maneira a preservar o meio ambiente local.

\subsection{Tipos de depósitos para rejeitos radioativos}

De acordo com IAEA, os repositórios são classificados em duas categorias principais: Próximos à superfície e geológicos.

Repositórios próximos à superfície podem ser simplesmente uma trincheira ou uma construção mais sofisticada, com barreiras de engenharia de concreto, etc. Têm sido utilizados em diversos países, entre os quais Inglaterra (Drigg), França (La Manche e Centre de L'aube), Canadá (Irus), Japão (Rokkasho), Brasil (CGP), Espanha (El Cabril), Suécia (Oskarshamn) e Estados Unidos (Barnwell), para a deposição de rejeitos do grupo 2.1 , sendo que o estabelecimento de um período de controle institucional é bastante importante, tendo em vista os riscos de intrusão a longo prazo.

Repositórios geológicos devem situar-se a uma distância da superfície, de forma a eliminar os riscos de intrusão humana, animais ou contato dos rejeitos com plantas. São classificados em três categorias: Cavidades especialmente feitas pelo homem (Finlândia); Minas abandonadas (Alemanha); e Cavidades naturais. Este tipo de repositório aplica-se preferencialmente aos rejeitos dos grupos 2.2 e 3 , da classificação da IAEA.

\subsection{Disposição geológica dos rejeitos de alto nível}

O termo "deposição geológica" refere-se à deposição de rejeitos radioativos sólidos em uma instalação subterrânea e, em uma formação geológica estável (geralmente várias centenas de metros abaixo da superfície), de modo a proporcionar um isolamento a longo prazo dos radionuclídeos da biosfera (Heilbron Filho et al. 2017). A deposição geológica foi concebida como um método para armazenar os tipos mais perigosos de rejeitos radioativos, incluindo o combustível irradiado de reatores e demais rejeitos de alto nível.

Os rejeitos a serem armazenados em uma instalação específica de deposição geológica devem ser determinados pela política e estratégia nacionais existentes. Os planos atuais de deposição geológica em vários países preveem que a instalação de deposição seja desenvolvida em diversas e diferentes etapas, abordagem que envolve estudos sobre: a avaliação do conhecimento científico necessários e existentes; dados técnicos relevantes do sistema de deposição; avaliação de possíveis sítios; desenvolvimento do conceito de deposição; estudos iterativos para o projeto e avaliação de segurança com o objetivo de progressivamente melhorar os dados; revisões técnicas e regulatórias existentes; consultas públicas; e decisões políticas.

Considerações sobre variedades de opções para o projeto e gerenciamento operacional de uma instalação de deposição geológica deve ter flexibilidade, sobretudo: a possíveis novas informações técnicas sobre a área; aos possíveis avanços na gestão de rejeitos adotadas por outros países; ao avanço de novas tecnologias de materiais, permitindo assim considerar os aspectos sociais, econômicos e políticos, que evoluem ao longo do tempo.

Em geral, são identificados três períodos associados ao desenvolvimento, operação e encerramento de uma instalação de deposição geológica: pré-operacional, operacional e pós-encerramento. Diversas atividades terão lugar durante esses períodos e algumas podem ser realizadas em graus variados ao longo de toda ou parte da vida útil da instalação:

- O período pré-operacional inclui a definição do conceito do depósito, a investigação do local e confirmação, avaliações de impacto ambiental, seleção de sítios, estudos de projeto e desenvolvimento dos modelos e aspectos de segurança operacional e pós-encerramento necessários para obter a autorização para 
prosseguir com a construção da instalação e das operações iniciais;

- O período operacional começa quando o rejeito é recebido pela primeira vez na instalação. A partir desse momento, a exposição à radiação pode ocorrer como resultado das atividades de gerenciamento, e estas devem estar sujeitas ao controle, de acordo com os requisitos de proteção contra radiações e segurança estabelecidos pelas normas de cada país. Neste período, programas de monitoramento e teste, com base nos quais a decisão de fechar a instalação de deposição geológica ou continuar são tomadas, assim como os principais aspectos pós-encerramento são desenvolvidos. Durante o período operacional, as atividades de construção podem ocorrer ao mesmo tempo da colocação dos rejeitos e encerramento de outras partes da instalação, período que pode incluir: Atividades para recuperação de rejeitos, se considerado necessário, antes do encerramento, atividades após a colocação dos rejeitos e o fechamento final com a devida vedação da instalação;

- O período pós-encerramento começa no momento em que todas as rotas de acesso à superfície são seladas. Após o encerramento, a segurança da deposição geológica é assegurada por meios passivos inerentes às características do sítio, das instalações e dos embalados dos rejeitos. Contudo, controles institucionais, incluindo algum monitoramento, podem continuar de maneira a garantir a segurança pública, por exemplo.

A segurança de uma instalação de deposição geológica, após o encerramento, depende de uma combinação de características do sitio, bem como da qualidade do projeto da instalação, dependendo também dos embalados utilizados e da implementação adequada do projeto, além da participação de profissionais competentes no planejamento, localização, fase de projeto e na implementação do mesmo.

Deposição geológica significa garantir o nível de segurança e qualidade necessários ao desenvolvimento do sistema de deposição de forma integrada, com base em uma sólida compreensão científica, boa engenharia, aplicação de sólidos princípios técnicos e gerenciais, e avaliações de segurança completas e robustas, e com a aplicação de garantia da qualidade (QA) para todos esses elementos.

O sistema de deposição geológica que compreende as instalações de deposição e o ambiente geológico em que o depósito está localizado é desenvolvido em diferentes etapas, em que a compreensão científica do sistema de deposição é feita de forma progressiva. Em cada etapa, a avaliação da segurança é importante para avaliar o nível de compreensão do sistema de deposição e para avaliar as incertezas associadas.

A base para essa compreensão do sistema de deposição e dos argumentos chaves para garantir a sua segurança e o reconhecimento das incertezas existentes e não resolvidas, devem ser incorporadas num relatório de análise de segurança, o qual inclui uma coleção de argumentos e evidências para demonstrar a segurança da instalação, que são desenvolvidos em conjunto com a progressão da construção da instalação. Esta abordagem fornece uma base para decisões relativas ao desenvolvimento, operação e encerramento da instalação, e permite a identificação de áreas em que a atenção precisa se concentrar para melhorar ainda mais a compreensão desses aspectos que influenciam a segurança do sistema de deposição geológica.

Portanto, a deposição geológica, como conceito, engloba uma variedade de opções, incluindo deposição especialmente em minas, em instalações construídas de engenharia e deposição em poços profundos. Sua característica principal é a profundidade da área, geralmente localizada a centenas de metros abaixo do nível do solo. Isso contrasta com a deposição próxima à superfície, situada um pouco abaixo do nível do solo (dezenas de metros), ou até mesmo acima da superfície. A seleção de local para construção de depósitos para rejeitos de alto nível, de acordo com Silva et al (2015), é um processo complexo que envolve também uma série de etapas e um conjunto de ferramentas, entre as quais: modelagem computacional, o uso de sistemas de informações georreferenciadas (SIG), entre outros.

\subsection{Principais documentos sobre 0 tema e os princípios da IAEA}

Os principais requisitos de segurança estabelecidos pela IAEA para a deposição geológica de rejeitos radioativos de alto nível de radiação estão previstos no WS-R-4 (IAEA 2006), derivado de outras documentações importantes: "Fundamentos Gestão de Segurança - Princípios dos rejeitos radioativos (Série Segurança No. 111. 1995); e Proteção contra Radiação e Segurança das Fontes de Radiação (Série Segurança No 120. 1996), são considerados os principais documentos da IAEA 
Tabela 2. Princípios gerencial dos rejeitos radioativos. (IAEA 1995).

\begin{tabular}{|c|c|c|}
\hline Princípio & Proteção & Características \\
\hline 1 & $\begin{array}{l}\text { Proteger a saúde } \\
\text { humana }\end{array}$ & $\begin{array}{l}\text { Atenção deve ser dada aos caminhos/vias de exposição do homem, os } \\
\text { princípios básicos de radioproteção também se aplicam a gerencia de } \\
\text { rejeitos, risco a longo prazo-incertezas }\end{array}$ \\
\hline 2 & $\begin{array}{l}\text { Proteger o meio } \\
\text { ambiente }\end{array}$ & $\begin{array}{l}\text { Manter as emissões de rejeitos em todas as etapas as menores possíveis, } \\
\text { concentrar e conter ao invés de dispersar e diluir, reciclagem, preservação } \\
\text { dos recursos naturais, riscos para outras espécies }\end{array}$ \\
\hline 3 & $\begin{array}{l}\text { Proteger além das } \\
\text { fronteiras do país }\end{array}$ & $\begin{array}{l}\text { Princípio ético, importância de se adotar regulamentos e recomendações } \\
\text { internacionais, intercâmbio de informações, etc }\end{array}$ \\
\hline 4 & $\begin{array}{l}\text { Proteger as gerações } \\
\text { futuras }\end{array}$ & $\begin{array}{l}\text { Aplicação do princípio de múltiplas barreiras de segurança, levar em } \\
\text { consideração a possibilidade futura }\end{array}$ \\
\hline 5 & $\begin{array}{l}\text { Estabelecer, no país, } \\
\text { um sistema legal } \\
\text { apropriado }\end{array}$ & $\begin{array}{l}\text { Deve haver uma separação entre a atividade operadora e fiscalizadora, } \\
\text { garantia de continuidade de responsabilidade a longo prazo }\end{array}$ \\
\hline 6 & $\begin{array}{l}\text { Minimizar a geração } \\
\text { de rejeitos }\end{array}$ & $\begin{array}{l}\text { Em todas etapas, tanto em volume quanto em atividade, reciclagem, } \\
\text { segregação adequada }\end{array}$ \\
\hline 7 & $\begin{array}{l}\text { Não transferir ônus } \\
\text { indevidos às gerações } \\
\text { futuras }\end{array}$ & $\begin{array}{l}\text { Princípio ético, a geração que se beneficiou da prática que de a solução } \\
\text { adequada, entretanto algumas atribuições podem ser deixadas para as } \\
\text { gerações futuras com o estabelecimento de um período de controle } \\
\text { institucional do depósito, informação da localização do depósito e do } \\
\text { inventário deve ser deixada de forma clara e segura para as gerações futuras }\end{array}$ \\
\hline 8 & $\begin{array}{l}\text { Levar em } \\
\text { consideração a } \\
\text { interdependência } \\
\text { entre geração e } \\
\text { gerência de rejeitos }\end{array}$ & Pré-tratamento, tratamento, acondicionamento, transporte, deposição final \\
\hline 9 & $\begin{array}{l}\text { Garantir a segurança } \\
\text { de instalações de } \\
\text { gerenciamento de } \\
\text { rejeitos }\end{array}$ & $\begin{array}{l}\text { Segurança em todas as etapas, seleção de local, operação, deposição final, } \\
\text { treinamento/qualificação das pessoas envolvidas }\end{array}$ \\
\hline
\end{tabular}

sobre questões de segurança e licenciamento de depósitos geológicos.

Conforme IAEA (1995), a gerência responsável pelos rejeitos radioativos está fundamentada em nove princípios (Tab. 2).

Atualmente, a estratégia adotada para atingir esses objetivos é concentrar-se em conter todos os tipos de rejeitos radioativos existentes e isolá-los da biosfera, que é aquela parte do ambiente que normalmente é habitado por organismos vivos e todos os elementos acessíveis aos seres humanos ou usada por estes, incluindo águas subterrâneas, águas superficiais e recursos marinhos, entre outros.

\section{Materiais e métodos}

Em termos metodológicos, o trabalho incorporou as principais referências bibliográficas da literatura internacional, sobretudo aquelas produzidas pela IAEA, considerados documentos-chaves acerca do tema, bem como a Lei de Rejeitos, que aponta a necessidade de se iniciar os estudos para a seleção de um depósito geológico no Brasil. A análise desses documentos subsidiou a elaboração das diretrizes gerais sobre a proteção radiológica, avaliação e requisitos de segurança em relação aos depósitos geológicos que podem ser utilizados para subsidiar a elaboração das normas necessárias para a deposição geológica até então não existentes. O fluxograma da figura 1 resume a metodologia adotada.

\section{DISPOSIÇÃO GEOLÓGICA DE REJEITOS RADIOATIVOS}

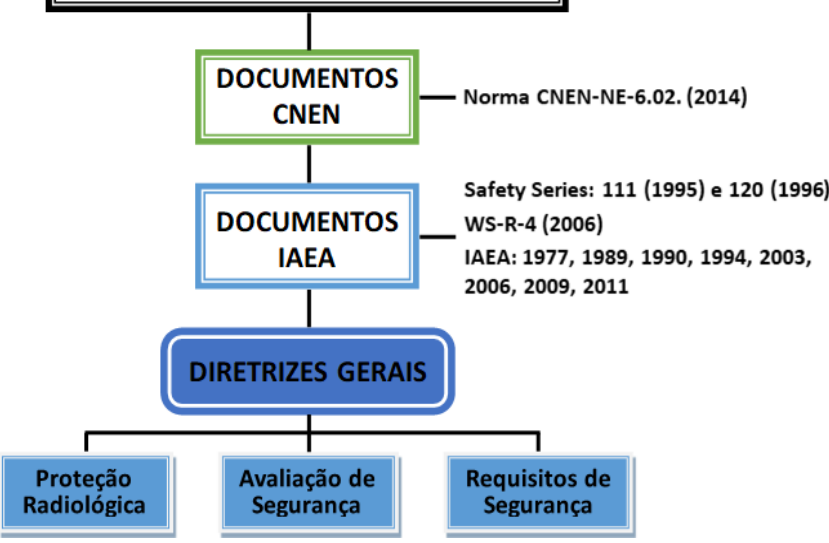

Figura 1. Fluxograma metodológico de desenvolvimento do trabalho

\begin{tabular}{c|c|c|c|c|c|}
\hline (C) Terrae Didat. & Campinas, SP & v.14 & p. $196-206$ & jul./set. 2018 \\
\hline \multicolumn{4}{|c|}{200}
\end{tabular}




\section{Diretrizes para a disposição geológica de rejeitos radioativos de alto nível}

Os repositórios geológicos têm sido a solução mais utilizada em países onde o gerenciamento dos rejeitos radioativos de alto nível são produzidos, sendo um recurso amplamente discutido nas conferências da IAEA e EURADWASTE, cujas conclusões definiram que os repositórios geológicos profundos são até o momento a medida mais segura e viável para a deposição segura dos rejeitos de alto nível.

Repositórios geológicos profundos constituem um repositório escavado entre $350 \mathrm{~m}$ ou $500 \mathrm{~m}$ abaixo da superfície, dentro de um ambiente geológico estável, o qual permita evitar durante muito tempo a contaminação da biosfera com o radionuclídeo radioativo (IAEA 2003). $\mathrm{O}$ isolamento neste tipo de deposição garante a segurança até que os produtos de fissão nuclear se desintegrem e atinjam níveis de radiação aceitáveis, ação que implica uma combinação de medidas, incluindo a forma do rejeito, pacote dos rejeitos, selos de engenharia e características geológicas adequadas para fornecer um alto nível de isolamento e contenção de longo prazo, sem necessidade de manutenção futura. Os recursos devem inibir ao máximo o movimento dos radionuclídeos no ambiente externo ao repositório, garantindo a segurança futura do meio ambiente e dos seres humanos (IAEA 1990), detalhes que são essenciais para garantir a segurança futura dos repositórios geológicos.

A seguir, apresentamos as diretrizes sobre os principais aspectos relevantes em relação à deposição dos rejeitos radioativos de alto nível: Proteção Radiológica, Avaliação e Requisitos de Segurança.

\subsection{Proteção radiológica}

A Proteção Radiológica abrange um conjunto de medidas que visam proteger o ser humano e seus descendentes contra possíveis efeitos indesejados causados pela radiação ionizante (CNEN 2005). Seu objetivo principal é evitar a exposição desnecessária dos indivíduos à radiação ionizante (Xavier et al. 2014).

\subsubsection{Proteção radiológica durante o período operacional do depósito}

Os requisitos de proteção radiológica para o período operacional de uma instalação de deposição geológica e os critérios de segurança relacionados são os mesmos aplicados para qualquer instalação nuclear licenciada, estabelecidos nas normas básicas de segurança de cada país.

Em termos de proteção radiológica, os rejeitos estão sob controle em uma instalação de deposição geológica durante o período operacional, já que as condições de segurança de seu armazenamento podem ser verificadas, as exposições podem ser controladas e ações, se necessário, podem ser tomadas.

Os aspectos de engenharia e meios práticos para alcançar a proteção são bem conhecidos, embora o uso subterrâneo de uma instalação de deposição geológica requer considerações específicas, cujo principal objetivo é garantir que as doses de radiação sejam tão baixas quanto razoavelmente exequiveis levando em consideração os fatores econômicos e sociais. Uma necessária, embora não suficiente condição, é que todas as doses devem ser mantidas dentro dos riscos aceitos.

A otimização da proteção (isto é, garantir que as doses de radiação sejam tão baixas como razoavelmente exequíveis, levando-se em consideração os aspectos sociais e econômicos) é necessária e deve ser considerada no projeto da instalação e no planejamento de operações acima e abaixo da superfície. Considerações relevantes incluem: a separação dos rejeitos dos resíduos de mineração, por ventura existentes e originários da escavação, o uso de controle remoto em equipamento de manuseio e equipamentos blindados para colocação de rejeitos, quando necessário; o controle do ambiente de trabalho, reduzindo o potencial de acidentes e suas consequências; e a minimização das necessidades de manutenção em áreas supervisionadas e controladas. A contaminação deve ser controlada e evitada na medida do possível.

Nenhuma liberação, ou apenas liberações muito pequenas de radionuclídeos podem ocorrer (como pequenas liberações de radionuclídeos gasosos) e nenhuma dose significativa para o público é permitida durante o funcionamento normal de um sistema de deposição geológica, incluindo a etapa de pós-fechamento.

Para uma instalação de deposição geológica, assim como para qualquer outra instalação nuclear em operação, é necessário um programa operacional de proteção contra radiações, a fim de garantir que as doses aos trabalhadores durante as operações normais sejam controladas e que os 
limites de dose sejam respeitados.

Além disso, são necessários planos de contingência para lidar com acidentes e incidentes, bem como para garantir que nesses casos as doses de radiação consequentes sejam controladas na medida do possível, com respeito aos devidos níveis relevantes de referência de emergência estabelecidos em norma.

As doses e riscos associados ao transporte dos rejeitos radioativos para dentro da instalação de deposição geológica devem ser gerenciados da mesma forma que as doses e os riscos associados ao transporte de outros materiais radioativos.

A segurança do transporte de rejeitos para a instalação de deposição geológica deve ser alcançada, cumprindo-se os requisitos estabelecidos pela CNEN em sua norma de segurança para o transporte de material radioativo (CNEN-NE-5.01).

\subsubsection{Proteção radiológica no período pós- fechamento}

A proteção da saúde humana e do ambiente no período pós-encerramento do repositório geológico deve apresentar as seguintes características:

- Objetivo. As instalações de deposição geológica devem ser localizadas, projetadas, construídas, operadas e fechadas para que a proteção no pós-fechamento seja otimizada, com os fatores sociais e econômicos sendo levados em consideração, com garantia razoável que as doses ou riscos para os membros do público a longo prazo não excederão a dose ou o nível de risco que foi usado como restrição de dose para o projeto;

- Critérios. O limite de dose para os membros do público de todas as práticas não deve ultrapassar o limite de dose efetiva de $1 \mathrm{mSv}$ em um ano, e este risco equivalente não deve ser excedido no futuro. Para cumprir com este limite de dose, uma instalação de deposição geológica (considerada como uma única fonte) deve ser projetada para que a dose média estimada ou risco médio para membros do público que possam estar expostos no futuro, como resultado das atividades envolvendo a instalação de disposição, não excedam a um valor de restrição de dose de $0,3 \mathrm{mSv} / \mathrm{ano}$ ou um risco equivalente a $10^{-5}$ por ano.

\subsubsection{Preocupações ambientais não radiológicas}

A avaliação dos impactos ambientais não-radiológicos, tais como os que ocorrem durante o período operacional, relacionados ao tráfego, barulho, amenidades visuais, perturbação dos habitats naturais, restrições ao uso da terra e fatores econômicos, estão fora do escopo das recomendações da CNEN, daí a importância de se fazer um termo de referência com o IBAMA.

Estimativas de doses devido à migração futura de radionuclídeos de uma instalação de deposição geológica são indicadores para a proteção das pessoas. As estimativas de dose para humanos devem levar em conta uma série de possíveis caminhos de transferência ambiental. Adicionalmente, indicadores e comparações, com estimativas das concentrações e fluxos de contaminantes e sua comparação com as concentrações e fluxos de radionuclídeos de ocorrência natural, também podem ser valiosos para indicar um nível de proteção ambiental global a longo prazo, de maneira independente a suposições sobre hábitos humanos. Outros fatores a serem considerados podem incluir a proteção dos recursos hídricos subterrâneos e a sensibilidade ecológica do ambiente em que os contaminantes podem ser liberados.

A instalação deve ser avaliada de acordo com regulamentos nacionais e/ou outros regulamentos específicos. Os fatores que devem ser considerados incluem o conteúdo químico, físico ou biológico dos rejeitos passíveis de liberação, de maneira a garantir a proteção de recursos de águas subterrâneas e a sensibilidade ecológica do meio ambiente.

\subsection{Requisitos de segurança}

Os requisitos de segurança são a garantia que o depósito geológico não irá impactar negativamente o meio ambiente e, consequentemente, a população local e os indivíduos chamados ocupacionais, que são aqueles que estarão envolvidos nas diversas etapas da seleção, construção, operação e encerramento do depósito geológico e que são treinados para trabalhos envolvendo exposição à radiação.

Considerando o planejamento para a construção de instalações de deposição geológica, os requisitos estão estabelecidos em três rubricas: o quadro jurídico e organizacional, a abordagem de segurança e os princípios do projeto de segurança.

Os princípios do projeto de segurança serão abordados em um artigo posterior. 


\subsubsection{Quadro jurídico e organizacional}

\section{Requisitos relacionados à responsabilidade do governo}

O governo é obrigado a fornecer uma base legal jurídica e organizacional dentro de uma instalação de disposição geológica, relacionada com sua localização, projeto, construção, operação e fechamento. Essa base deve incluir a definição das etapas no desenvolvimento e licenciamento da instalação, incluindo a clara alocação de responsabilidades, a obtenção de recursos financeiros e outros recursos importantes (humanos, por exemplo), bem como a provisão de funções reguladoras independentes.

Tal base é um dos princípios básicos da gerência de rejeitos radioativos, bem como também está estabelecida nos termos da Convenção Conjunta sobre os rejeitos radioativos e combustível irradiado, da qual o Brasil é signatário. As principais questões que devem ser consideradas incluem:

- Definir a política nacional para o gerenciamento a longo prazo de rejeitos radioativos de diferentes tipos;

- Definir responsabilidades legais, técnicas e financeiras claramente das organizações envolvidas no desenvolvimento da gestão de rejeitos e de instalações de deposição, incluindo instalações de disposição geológica;

- Garantir a adequação e a segurança das provisões financeiras;

- Definir o processo geral para o desenvolvimento, operação e fechamento das instalações de deposição, incluindo os requisitos legais e regulamentares (condições da licença) em cada etapa e nos processos de tomada de decisão, incluindo ainda todas as partes interessadas;

- Garantir que o conhecimento técnico e científico necessário permaneçam disponíveis tanto para o operador quanto para o regulador;

- Determinar as responsabilidades legais, técnicas e financeiras para quaisquer arranjos institucionais previstos no pós-fechamento, incluindo o monitoramento e garantindo assim a segurança da deposição dos rejeitos armazenados, bem como dos indivíduos ocupacionalmente expostos, da população e do meio-ambiente.

\section{Requisitos relacionados à autoridade reguladora}

O órgão regulador deve estabelecer os requisitos regulamentares para a construção de instalações de deposição geológica e deve estabelecer os procedimentos e requisitos de segurança para as várias etapas do processo de licenciamento, incluindo as condições de operação e encerramento da instalação de deposição geológica, incluindo todas as atividades necessárias para certificar que todas as condições estabelecidas previamente foram atendidas.

Normas gerais para a proteção da saúde humana e do ambiente geralmente são estabelecidas em uma política nacional ou em legislação especifica, como é o caso do Brasil. O órgão regulador desenvolve requisitos regulamentares específicos para a instalação, com base na política nacional e levando em conta o objetivo e critérios estabelecidos. O órgão regulador é o responsável pela orientação sobre a interpretação da legislação nacional e regulamentadora para o operador e para as partes interessadas, de maneira a garantir que os requisitos regulamentares são apropriados e praticáveis. O corpo técnico do órgão regulador também realizará pesquisas, adquirirá capacidades de avaliação independentes e deve participar da cooperação internacional, conforme necessário, de maneira a cumprir suas funções corretamente.

O órgão regulador documentará os procedimentos que ele usa para avaliar a segurança da instalação, os procedimentos que os operadores devem seguir no contexto de licenciamento e pré-licenciamento, incluindo pedidos de licenças e os procedimentos que se aplicam à revisão de documentação apresentada, de maneira a avaliar a conformidade com os regulamentos e com os requisitos pré-estabelecidos.

\section{Requisitos relacionados ao operador do depósito geológico}

O operador de uma instalação de disposição geológica será responsável por sua segurança. $\mathrm{O}$ operador deve realizar avaliações de segurança e apresentar a documentação sobre a análise, deve realizar todas as atividades necessárias para localização, projeto, construção, operação e encerramento, em conformidade com a regulamentação e requisitos estabelecidos dentro da infraestrutura legal nacional.

O operador é responsável pelo desenvolvimento de modelos de análise de segurança de maneira demonstrar sua segurança, de forma consistente com os requisitos do órgão regulador. Esta tarefa é realizada levando-se em consideração as características e quantidades dos rejeitos radioativos a serem 
depositados, das características do sitio, ou dos locais disponíveis, das técnicas de mineração e engenharia disponíveis e da infraestrutura legal nacional e os requisitos regulamentares estabelecidos.

O operador é também responsável pela preparação de um caso de segurança completo, com base no qual decisões sobre o desenvolvimento do projeto, operação e encerramento da deposição geológica serão feitas.

O operador é responsável pela realização ou comissionamento de toda pesquisa e desenvolvimento necessários para garantir e demonstrar que o planejado e as operações técnicas podem ser realizadas de forma prática e segura, incluindo todas as pesquisas necessárias para investigar, compreender todos os processos envolvidos na segurança da instalação.

O operador também é o responsável pela realização de todas as investigações necessárias do sitio escolhido e ou dos demais sítios investigados para fornecer dados para as avaliações de segurança.

O operador deve estabelecer as especificações técnicas que devem ser justificadas pela avaliação de segurança, para garantir que a instalação de deposição geológica seja desenvolvida de acordo com o caso de segurança. Isso inclui critérios de aceitação de rejeitos e outros controles e limites a serem aplicados durante a construção, operação e fechamento. O operador deve manter todas as informações relevantes para o caso de segurança, manter todos os registros de inspeção que demonstram a conformidade com os requisitos regulatórios e com a própria especificação do operador. Tais informações e registros serão mantidos pelo menos até o momento em que as informações são exibidas como sendo substituídas ou até que a responsabilidade pela instalação seja passada para outra organização, como pode ocorrer no caso do encerramento.

O operador deve cooperar com o órgão regulador e fornecer toda a informação que os reguladores solicitarem e exigirem para fins de licenciamento. A necessidade de preservar os registros para longos períodos de tempo deve ser levada em consideração quando da escolha e seleção do formato e mídia a ser usada para os registros.

\subsubsection{Avaliação de segurança de depósitos geológicos (abordagem de segurança)}

Requisitos relativos à importância da segurança no processo de desenvolvimento do depósito

Ao longo do desenvolvimento de uma instalação de deposição geológica, a compreensão adequada da relevância e implicações para a segurança das opções disponíveis devem ser avaliadas pelo operador com o objetivo final de garantir um nível otimizado de segurança operacional e de pós-encerramento.

As instalações de deposição geológica são desenvolvidas e operadas ao longo de um período de vários anos ou décadas. Assim, as principais decisões, como decisões sobre a localização, projeto, construção, operação e fechamento da instalação, deverão ser feitas durante o desenvolvimento do projeto. Neste processo, as decisões são tomadas com base nas informações disponíveis no momento, que podem ser de natureza quantitativas ou qualitativas e com base no grau de confiança das mesmas.

O desenvolvimento, operação e encerramento da instalação são limitados por fatores externos, como a política nacional e preferências e a disponibilidade de locais adequados e formações geológicas dentro das fronteiras nacionais para hospedar uma instalação adequada. No entanto, um nível adequado de confiança no local e na construção/operação deve ser garantido.

Em cada momento de decisão principal, as implicações para a segurança das opções disponíveis devem ser consideradas e levadas em conta. Assegurar a segurança operacional e no pós-encerramento é importante durante cada momento de decisão incluindo fatores relacionados a aceitação pública, custo, propriedade do sitio, infraestrutura e rotas de transportes.

Deve ser dada preferência a locais situados longe de minerais subterrâneos conhecidos, fontes geotermais e aquáticas, recursos existentes para reduzir o risco de intrusão humana no sitio e potencial para os usos da área circundante que estão em conflito com a instalação.

A segurança é considerada em cada etapa do processo de tomada de decisão para garantir que instalação está otimizada em todos os níveis.

\section{Requisitos relativos à segurança passiva}

O operador deve localizar, projetar, construir, operar e fechar a instalação de deposição de tal forma que a segurança pós-encerramento seja assegurada de modo passivo, o que significa que a segurança não depende de ações a serem realizadas após o encerramento da instalação.

O fornecimento de segurança passiva no período pós-encerramento implica em uma adequada gestão ativa da instalação no período anterior. 
A cessação da gestão significa que a instalação de deposição geológica, não apresenta mais risco de exposição à radiação, não necessitando de controles ativos.

Assim, devem existir barreiras naturais e projetadas (de engenharia) que proporcionem segurança no período pós-encerramento.

$\mathrm{Na}$ prática, controles institucionais, incluindo restrições ao uso da terra, podem ser mantidos mesmo após a instalação de deposição geológica ter sido fechada. Tais controles e monitoramento não são necessários para garantir a segurança da instalação; no entanto, eles podem ser considerados como medidas adicionais de garantia de segurança.

\section{Requisitos para uma compreensão adequada de construção de confiança na segurança}

O operador de uma instalação de deposição geológica deve ter compreensão das características, eventos e processos que influenciam o seu pós-fechamento durante períodos de tempo adequadamente longos, de modo que um nível suficiente de confiança na segurança seja alcançado.

A construção de confiança é facilitada através da identificação dos recursos e processos existentes no local e também pelas características, eventos e processos que podem ser prejudiciais para a segurança, sendo importante demonstrar que estão suficientemente caracterizados e entendidos. Toda incerteza, deve ser levada em consideração na estimativa de segurança.

O objetivo é garantir que existe um alto nível de confiança nos recursos e processos considerados durante o período requerido de contenção e isolamento dos rejeitos.

A demonstração exige que a segurança seja garantida e comprovada utilizando-se modelos robustos (ou seja, modelos cujo desempenho seja de baixa sensibilidade para os eventos e processos perturbadores mais importantes do sistema), e sejam apresentadas provas suficientes de sua viabilidade $\mathrm{e}$ eficácia, antes das atividades de construção começarem. A este respeito, a gama de possíveis eventos e processos perturbadores que é razoável incluir em tais considerações deve estar sujeita à aprovação por parte do órgão regulador. Essas considerações indicam haver compreensão de se tais eventos e processos poderiam levar a perda generalizada de funções de segurança do sistema de disposição.

Uma compreensão do desempenho do sistema de deposição e dos processos existentes evolui à medida que mais dados são acumulados e mais conhecimento científico é desenvolvido. No início do desenvolvimento do conceito, os dados e o nível de compreensão adquiridos devem proporcionar a confiança necessária para investir os recursos em futuras investigações. Antes do início da construção, durante a colocação e no encerramento, o nível de compreensão deve ser suficiente para confirmar os resultados do relatório de segurança e apresentar garantias de cumprimento dos requisitos regulamentares aplicáveis. Ao estabelecer esses requisitos, é importante reconhecer que existem múltiplos componentes de incerteza inerentes à modelagem de sistemas ambientais complexos e que inevitavelmente existem incertezas significativas associadas ao projeto e desempenho de um sistema de disposição geológica.

\section{Conclusões}

Este artigo abordou os principais aspectos de proteção radiológica e requisitos de segurança para o planejamento de instalações de disposição geológica, bem como os principais aspectos relacionados com a avaliação de segurança para a construção de depósitos geológicos. Além disso, os aspectos apontados evidenciam a importância de fortalecer a área de rejeitos radioativos da CNEN, sobretudo com vistas à elaboração das normas que são necessárias para a seleção, construção e licenciamento de depósitos geológicos, visando a correta disposição dos futuros rejeitos de alto nível de radiação produzidos pelas usinas nucleares brasileiras, e que estejam de acordo com as normas e recomendações internacionais.

\section{Agradecimentos}

Os autores agradecem aos revisores e editores pelas contribuições e enriquecimentos para melhorias do artigo, bem como à CNEN, pelo fomento à pesquisa, além de FAPERJ, CAPES e CNPq, importantes instituições de fomento à pesquisa no Brasil.

\section{Referências}

CNEN. 2005. NORMA CNEN-NN-3.01. Diretrizes Básicas de Proteção Radiológica. Resolução No 27. Publ.: D.O.U 06/01/2005.

CNEN. 2014. Norma CNEN NN 8.02. Resolução CNEN 168/14. Licenciamento de Depósitos de Rejeitos Radioativos de Baixo e Médio Níveis de 
Radiação. Resolução CNEN 168/14.

Heilbron Filho P.F.L., Perez Guerrero J.S., Heilbron M.C.P.L., Valeriano C.M., Silva, C. 2017. Radioactive Waste Management in Brazil Including Spent Fuel. In: B. Faybishenko, J. Birkholzer, D. Sassani, and P. Swift. orgs. International Approaches for Nuclear Waste Disposal in Geological Formations: Geological Challenges in Radioactive Waste Isolation - Fifth Worldwide Review. Berkeley: Lawrence Berkeley Nat. Lab. (LBNL), v. 1, p. 35-60. DOI: https://doi. org/10.2172/1353043.

IAEA. 1977. Site Selection Factors for Repositories of Solid High Level and Alpha Bearing Wastes in Geological Formations. Technical Reports Series No. 177. 76p.

IAEA. 1989. Safety Principles and Technical Criteria for the Underground Disposal of High Level Radioactive Wastes. IAEA Safety Series No. 99. 40p.

IAEA. 1990. Qualitative acceptance criteria for radioactive wastes to be disposed of in deep geological formations. IAEA TECDOC No. 560. 44p.

IAEA. 1994. Siting of Geological Disposal Facilities: a safety guide. IAEA Safety Series No. 111-G-4.1. 48p.
IAEA. 1995. Principles of Radioactive Waste Management: Safety Fundamentals. IAEA Safety Series No. 111S-F. 24p.

IAEA. 2003. Radioactive Waste Management Glossary: 2003 Edition. IAEA Non-serial Publications. 54 p.

IAEA. 2006. Geological Disposal of Radioactive Waste: Safety Standards. IAEA Safety Standards Series No. WSR-4. 49p.

IAEA. 2009. Classification of Radioactive Waste: General Safety Guide. IAEA Safety Standards Series No. GSG-1. 48p.

IAEA. 2011. Geological Disposal Facilities for Radioactive Waste: Specific Safety Guide. IAEA Safety Standards Series No. SSG-14. 104 p.

Silva C., Heilbron M.C.P.L., Heilbron Filho P.F.L. 2015. Site Selection of a Geological Repository for the Safe Disposal of High-Level Waste in the State of Rio de Janeiro. Rev. Int. Ciênc., 5:83-105. DOI: https://doi.org/10.12957/ric.2015.16615.

Xavier A.M., Moro J.T., Heilbron P.F.L. 2014. Princípios básicos de segurança e proteção radiológica. 4 ed. UFRGS/CNEN. 